\title{
The role of the psychiatrist in the care of women with breast cancer
}

\author{
M. J. Massie \\ Memorial Sloan-Kettering Cancer Center, New York, NY, USA.
}

\begin{abstract}
Psychiatric consultation and supportive psychotherapy provide many benefits for women with breast cancer including an opportunity to address existential, physical, emotional, social, psychosexual and relationship (family and others) concerns. The psychiatrist is a source of meaningful information and helps the patient challenge pessimistic thoughts and consider life, health, family and career priorities. The management of psychiatric symptoms and the treatment of side effects helps promote adherence to cancer treatment.
\end{abstract}

Keywords: Breast cancer; Psycho-oncologist; Psychotherapy

\section{Introduction}

In specialized breast centers, psychiatrists (psychooncologists) are integral members of the breast oncology team, sharing office and waiting room space with surgical, medical or radiation oncologists, participating in interdisciplinary teaching conferences and tumor board reviews and collaborating in patient care and research. The psychological and psychosocial aspects of breast cancer have received much attention over the last 30 years for many reasons, including the high prevalence and mortality of the disease, the psychological affects of surgery on an organ rich in meaning (body and self-image, sense of attractiveness, femininity, sexuality, nurturing capacity and reproduction), and the affects of long and arduous chemotherapy. All clinicians know that the diagnosis of breast cancer is a significant stressor for any woman, but women's emotional responses to being at high risk, to hearing the diagnosis of a life-threatening illness, to breast cancer treatment and to the post-treatment period vary considerably.

Correspondence to: Mary J. Massie, MD, Attending Psychiatrist, Memorial Sloan-Kettering Cancer Center, 1275 York Avenue, New York, NY 10021, USA. E-mail: massiem@mskcc.org

Publication date 31/05/05 BCO/252/2004/FO
The experienced breast oncologist has a good internal barometer for noting emotional responses that fall outside the range of normal and referring those women for psychiatric evaluation. The new house officer often asks what is the role of the psychiatric consultant in the care of patients with breast cancer? In our breast cancer center, patients who are referred are usually who fall in one of several groups.

\section{Women at high risk of developing breast cancer}

The patients at our center who consider a bilateral mastectomy and oophorectomy as risk-reducing surgeries usually have been evaluated by our clinical genetics service are extremely well informed about inherited breast cancer and the nature of these procedures as a result of consultations with surgeons, attending lectures or support groups, and speaking with women who have had these surgeries. However, many women benefit from psychiatric consultation as they are in the process of deciding whether or when to have prophylactic mastectomy. The psychiatrist's office is a private place for the patient to consider options that have both personal and family ramifications and to imagine both short-term and enduring emotional responses. With the goals of reducing the patient's anxiety to manageable levels, 
understanding whether the surgeon's recommendations and reassurance have been heard, and effectively reinforcing the information and advice provided by the surgeon and the genetic counselor, the psychiatrist reviews the woman's family history of breast and other cancers, her psychiatric history and current mental status, and her history of and satisfaction with any previous plastic surgeries. The presence of anxiety, depression or other psychiatric disorders, and the presence and adequacy of social support, body and self-image concerns, self-esteem, decisionmaking style and both adaptive and maladaptive coping styles are reviewed.

Women consider the ramifications of risk-reducing surgeries in terms of emotional, social, sexual and marital functioning, and adaptation to and the timing of surgery in relationship to future pregnancies or other important family needs and plans. The psychiatrist serves as a sounding board, offers support (not personal opinion), and assists the patient in effective decision-making. The psychiatrist queries whether anyone may be unduly influencing the patient's decision and treats psychiatric disorders, such as depression, anxiety or insomnia that may be affecting her decision-making. Occasionally (but very rarely), the psychiatrist recommends postponing the consideration of surgery until marital or relationship disagreements can be better understood or resolved, or until a significant concurrent life event is completed and the patient can give her full attention to the consideration of her health.

The psychiatrist encourages the woman to invite her husband or partner to a session, so the fears and questions of the partner can be aired and answered. Having the partner consider his or her response to the proposed surgery is described by most women as an extremely important part of the consultation. Women often request psychiatric consultation to discuss how to prepare small or pre-adolescent children for their absence from the home for surgery and/or how to describe surgery in age appropriate terms.

\section{Women who have been diagnosed with breast cancer and are considering treatment options}

The second group of women commonly referred for psychiatric consultation is composed of those who are so overwhelmed after hearing the diagnosis of breast cancer that they are unable to make reasonable and timely decisions for treatment and to comply with treatment recommendations. Although most women are able to make decisions, even under the overwhelming circumstance of having been told 'you have breast cancer,' those who seem emotionally paralyzed benefit from psychiatric consultation. Sometimes these women describe feeling pressured 'to make the most important decision of my life,' with multiple conflicting medical opinions or biased opinions from fearful family members and friends who are armed with out-dated information and personal prejudice. The psychiatrist views these referrals as urgent since decision-making cannot be endlessly delayed. One to two sessions is often all that is required to help a woman review her understanding of proposed treatment options, to mentally organize the information that she has been told, and to formulate any remaining questions that she has for her surgeons and radiation or medical oncologists. The psychiatrist corrects misperceptions and reinforces information that has been correctly understood. The psychiatric consultation room is a quiet place for a woman to 'sort through' her fears with an experienced professional and to 'try on' all considerations as she seeks the best treatment fit.

\section{Women who are receiving breast cancer treatment}

Oncologists should consider a referral for psychiatric consultation when the patient's clinical course is complicated by psychiatric symptoms or overwhelming personal stressors. Among the psychiatric disorders of most concern, anxiety and depression are the most frequent and often the most disabling. Some women who are being treated for a mood or an anxiety disorder wisely request referral to a psychiatrist who specializes in working with women breast cancer because of their fear that they are 'at risk' for a relapse of their psychiatric disorder or a worsening of their symptoms because of the stress of cancer treatment. Others will be delighted to learn that psychiatrists are available to monitor mood, anxiety and cognition while one undergoes stressful treatment including treatment with drugs (such as dexamethasone) that can cause insomnia, irritability, anxiety and depression. Women who may be at risk for difficulty in adjusting to a breast cancer treatment are those who are very young, elderly, pregnant or nursing at the time of diagnosis, single or alone, or in a nonsupportive relationship. Additionally, women who are adjusting to multiple losses or who are managing multiple life stresses during cancer treatment benefit from psychiatric consultation.

The time to ask about psychiatric disorders, psychotropic medication use and concurrent life stressors is at the first visit when the oncologist reviews the patient's medical history. Sometimes patients report that they did not want 'to bother' their oncologist with their psychiatric problems or that they wanted to keep their oncologist 'focused' on their cancer so 
they did not mention their psychiatric condition unless asked. These patients are relieved to learn that their oncologist views their panic disorder or, for example, bipolar disorder as important and treatable as their hypertension or diabetes. Patients who have a history of suicide attempt, psychiatric hospitalization, bipolar disorder or mood swings, recurrent depression, panic disorder, insomnia or substance or alcohol abuse should be referred for psychiatric consultation. Patients who are depressed or say 'I don't think I can do this cancer treatment' or 'I am not functioning' or those whose family members report 'She has just collapsed emotionally' likewise should be referred. Periodically throughout cancer treatment, all patients should be asked 'How is your mood (your spirits) holding up throughout all this?' Those who indicate that they are distressed, depressed or struggling to maintain only a low level of functioning need help.

A psychiatric management approach that combines psychological support in either individual or group settings and the use of antidepressants, anxiolytics and hypnotics helps reduce distressing symptoms to tolerable levels so that the patient can comply with cancer treatment recommendations and to 'live better' during treatment.

The psychiatrist, medical oncologist and sexual health specialists work in concert to address the sexual issues that commonly complicate breast cancer treatment. The major sexual issues in women who are being or have been treated for breast cancer are decreased libido (desire); decreased vaginal lubrication; painful sex; decreased pleasure in sex; embarrassment about scars, implants, alopecia, lymphedema, weight gain (loss); concern that her partner will injure her during intercourse and fear of resuming sex after cancer treatment. Frequently, the psychiatrist is asked to prescribe and regulate the dose of the antidepressants (sertraline, paroxetine or venlafaxine) commonly used to treat vasomotor menopausal symptoms (hot flashes).

Some women need advice about whether, when or what to tell an employer, colleagues, friends, a new date or new sexual partner about their breast cancer treatment.

Many women have questions about stress as a factor in the incidence or progression of breast cancer and many incorrectly believe scientists have conclusively demonstrated a one-to-one correlation between stress and cancer. Some patients choose to consider effective stress-reduction techniques as a lifestyle (not cancer preventing) option.

\section{Summary}

In summary, psychiatric consultation and supportive psychotherapy provides many benefits for women with breast cancer including an opportunity to address existential, physical, emotional, social, psychosexual and relationship (family and others) concerns. Psychiatric consultation and the psychotherapy that may follow is an opportunity to express emotions, gain support, and manage anxiety, fear and depression. The psychiatrist is a source of meaningful information and helps the patient challenge pessimistic thoughts and consider life, health, family and career priorities. The management of psychiatric symptoms and the treatment of side effects helps promote adherence to cancer treatment and the discussion of phase specific issues such as fear and increased anxiety at the end of cancer treatment or the feasibility of childbearing or child rearing with uncertainty about the future helps a woman make a transition from 'cancer patient' status to her usual life challenges, responsibilities and pleasures.

\section{Further reading}

1. Massie MJ, Greenberg D. Oncology. In: Levenson J (Ed.). APPI Textbook of Psychosomatic Medicine. Washington: American Psychiatric Press; 2004: 597-618.

2. Rowland $\mathrm{JH}$, Massie MJ. Issues in breast cancer survivorship. In: Harris JR, Lippman ME, Morrow M, Osborne CK (Eds). Diseases of the Breast, 3rd edition. Philadelphia: Lippincott, Williams and Wilkins; 2004: 1419-1452. 